\title{
A Mean-Value Model for Control of Homogeneous Charge Compression Ignition (HCCI) Engines
}

\author{
D. J. Rausen, A. G. Stefanopoulou \\ The University of Michigan
}

\begin{abstract}
A Mean Value Model (MVM) for a Homogeneous Charge Compression Ignition (HCCI) engine is presented. Using a phenomenological zero-dimensional approach with five continuous and three discrete states we first model the effects of the Exhaust Gas Recirculation (EGR) valve, the exhaust Rebreathing Lift (RBL), and the fueling rate on the state of charge in the cylinder at intake valve closing. An Arrhenius integral is then used to model the start of combustion, $\theta_{s o c}$. A series of simple algebraic relations that captures the combustion duration and heat release is finally used to model the state of charge after the HCCI combustion and the Location of Peak Pressure (LPP). The model is parameterized and validated using steady-state test data from an experimental engine at the General Motors Corporation.

The simple model captures the temperature, pressure, airto-fuel ratio, and inert gas fraction of the exhausted mass flow. This characterization is important for the overall HCCI dynamics because the thermodynamic state (pressure, temperature) and concentration (oxygen and inert gas) of the exhausted mass flow affect the next combustion event. The high dilution level in HCCI engines increases the significance of this internal feedback that generally exists to a smaller extent in conventional spark-ignition and compression-ignition internal combustion engines.
\end{abstract}

\section{INTRODUCTION}

The basis of Homogeneous Charge Compression Ignition (HCCI) engines is their fast and flameless combustion after an autoignition process of a homegeneous mixture. HCCI combustion achieves high fuel efficiency [10] with low NOx emissions due to the limited cylinder peak temperature (below $1700 \mathrm{~K}$ ). The timing of HCCI combustion is determined by mixture conditions, rather than the spark timing or the fuel injection timing that are used to initiate combustion in Otto and Diesel engines, respectively. Instead, controlled autoignition requires regulation of the mixture properties (temperature, pressure, and composition) at the Intake Valve Closing (IVC). Observations show that Variable Valve Timing (VVT) flexibility can provide control over the mixture conditions at IVC [5], [16]. For example, early Exhaust Valve Closing (eEVC) and late Intake Valve Opening (IIVO) enable internal EGR (iEGR) with high temperature trapped residuals, which also alleviates the preheating need [8]. To control hot residuals (internal Exhaust Gas Recirculation or iEGR), we consider in this paper an actuation technique called exhaust rebreathing, in which the exhaust valve is reopened during the intake stroke, as shown in Fig. 1. A conventional EGR valve allows control of the external Ex-

\footnotetext{
Funding for this work was provided by the General Motors Corporation under contract TCS-09026

Corresponding author, annastef@umich.edu,ph:(734)615-8461.
}

\author{
J-M. Kang, J. A. Eng, T-W. Kuo \\ General Motors Corporation
}

haust Gas Recirculation (eEGR) and thus the cold fraction of the inert mass trapped at IVC.

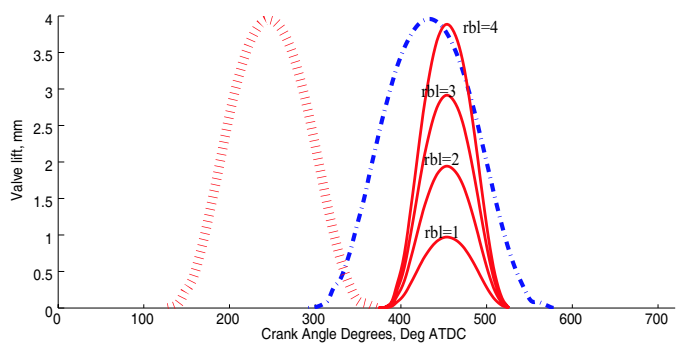

Fig. 1. Exhaust, intake and rebreathing valve profiles

Control synthesis and design requires a model that represents the effects of the valve actuators to the charge conditions and the HCCI combustion characteristics. Phenomenological crankangle-resolved HCCI combustion models have been developed originally in [10] and recently in [14]. Although these models are indispensable for understanding and simulating HCCI combustion, low-order models are necessary for real-time feedback and observer design. Efforts to extract information from the crankangleresolved models and control the peak cylinder pressure of propane-based HCCI combustion are presented in [15]. Input-output models based on system identification are shown in [2] and used for control of the combustion timing with dual-fuel. Papers that present experimental results with controlled HCCI combustion through in-cylinder pressure or ion current feedback have used decoupled (single-input single-output) control laws. In [11] three decentralized PI controllers for an inlet heater, fuel charge, and fuel octane ratio are used to regulate inlet temperature to 80 degrees, track IMEP commands, and regulate 50 percent burn timing (CA50) to a range of 3-8 degrees ATDC. The last control goal is the hardest to achieve. In two recent papers [1], [12], PI controllers are used for intake valve closing or negative overlap. The authors anticipate better results with modelbased tuning of the controller gains and coordination of all the available actuators.

To this end we develop in [13] a Mean Value Model (MVM) that captures the effects of the exhaust valve rebreathing lift $u_{r b l}$, the EGR valve $u_{e g r}$, and the gasoline fueling rate $W_{f}$ to the charge composition and combustion characteristics. Specifically, we consider as performance variables the in-cylinder air-to-fuel ratio (AFR) at the exhaust tailpipe and the crank angle of $50 \%$ fuel burned, 
$\theta_{C A 50}$ that approximates the location of peak pressure $\theta_{L P P}$. We assume the availability of a wide range, fast, heated Exhaust Gas Oxygen (EGO) sensor for AFR measurements in the exhaust manifold $A F R_{E G O}$. In summary, we develop the appropriate model for regulating AFR and $\theta_{C A 50}$ by coordinating the rebreathing valve lift $u_{r b l}$, and the EGR valve $u_{e g r}$ during step changes in fueling rate $W_{f}$ and constant engine speed $N$. The input-output signals and other important variables used in this paper are shown in Fig. 2.

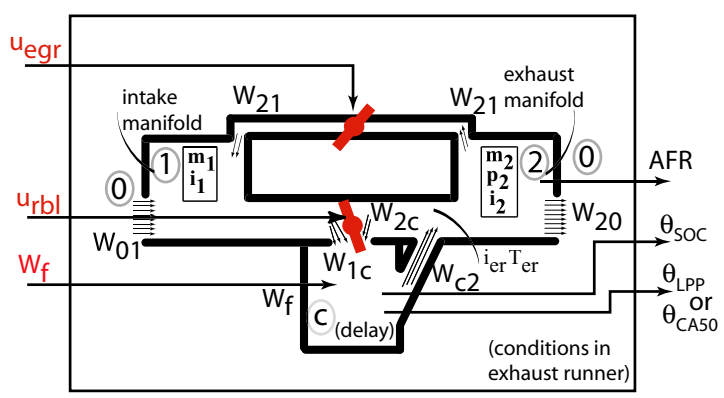

Fig. 2. Schematic diagram and notation for the mean value model.

\section{Model Structure}

The model presented here includes a physics-based parametrization of HCCI behavior. The MVM dynamical behavior is associated with (i) states representing the mass composition and pressures in the intake and exhaust manifold volumes and (ii) the delay between cylinder intake and exhaust processes. Cycle-average cylinder flows at temperatures $T_{1}$ and $T_{e r}$ enter the cylinder (volume $c$ ) from intake (volume 1) and exhaust manifold (volume 2), respectively, as shown in Fig. 2. Variables associated with ambient conditions are denoted with subscript 0 . Masses at volume $x$ are called $m_{x}$ and pressures are called $p_{x}$. To account for composition dynamics, inert gas fraction $i_{x}$ at volume $x$ is defined as

$$
i_{x}=\frac{\text { mass of inert gas in } x}{\text { total mass in } x}=\frac{m_{x}^{i}}{m_{x}}
$$

where $m_{x}^{i}$ is the mass of inert gas (combustion products) at volume $x$. Since the HCCI engine operates lean, both the flow through the EGR valve and the rebreathed residuals contain air. Mixture compositions therefore include three components: air, fuel, and inert gas.

The five model states defined by differential equations are shown in rectangular boxes. Flows are depicted according to the notation $W_{x y}$ where $x$ is the source of the flow and $y$ is the sink. The exhaust flow, $W_{c 2}$, from cylinder to exhaust is considered as a delayed sum of the flows into the cylinder. The other two delayed states correspond to the exhaust runner gas temperature, $T_{e r}$ and inert gas fraction, $i_{\text {er }}$.

The MVM is parameterized from steady-state test data collected from a single-cylinder engine described in Sec. III. The combustion process is considered as a series of causal algebraic relations whereby conditions at Intake Valve Closing (IVC) determine those at Exhaust Valve Opening (EVO). The combustion model provides the composition and temperature of the gas that is exhausted and fed back to the cylinder through eEGR and iEGR, representing internal feedback from the Manifold Filling Dynamics (MFD) through the HCCI combustion and back to the MFD. The integrated model, shown in Fig. 3 captures both the steadystate relations between the inputs and outputs and their dynamical evolution. The model is accurate for dynamics slower than the cycle period, or those below $\omega_{v}=\frac{2 N \pi}{120}$ $\mathrm{rad} / \mathrm{sec}$ where $\mathrm{N}$ is in $\mathrm{rev} / \mathrm{min}$.

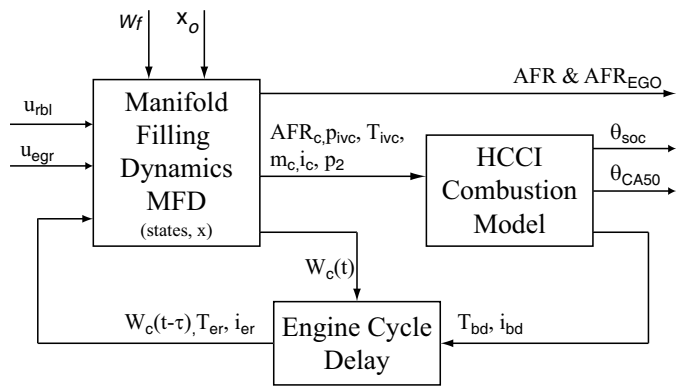

Fig. 3. The integrated mean value model, consisting of Manifold Filling Dynamics (MFD) and HCCI combustion model.

\section{EXPERIMENTAL SET-UP}

Dynamometer experiments used to parameterize the MVM were performed on a single-cylinder engine $(86 \mathrm{~mm}$ bore, $94.6 \mathrm{~mm}$ stroke, $0.55 \mathrm{~L}$ displacement, compression ratio $\mathrm{CR}=14$ ), at $1000 \mathrm{RPM}$ and a range of loads. The cylinder head includes four valves, a pent-roof combustion chamber, and a spark plug that was not activated during these experiments. A flat-top piston with valve cutouts enabled spin-free operation of the fully flexible intake and exhaust valves.

Sonic nozzles measured airflow, and two large plenums damped intake pressure pulsations. Plenums heaters maintained intake air temperature of $90^{\circ} \mathrm{C}$. Fuel flow was measured with a Pierburg PLU 103B flow meter. Exhaust gas recirculation (EGR), controlled manually via a ball valve, was mixed with the fresh air and fuel mixture upstream of the engine in the first intake plenum. External heat exchangers maintained air and water temperatures at $95^{\circ} \mathrm{C}$.

A Kistler 6125 pressure transducer located at the rear of the combustion chamber along the axis of the pent-roof was amplified with a Kistler 504E charge amplifier. Crankshaft position was measured with a Dynapar crankshaft encoder, and a hall-effect sensor provided camshaft location. A Sensotec strain gage transducer provided intake manifold pressure, which calibrated the cylinder pressure reading at the bottom of the intake stroke. Cylinder pressure data was recorded using the MTS-DSP Advanced Combustion Analysis Program (ACAP) that calculates performance parameters such as Peak Pressure and location of peak pressure in real time. 
A simple filling model using partial pressure of the intake mass and the exhaust gas temperature in the exhaust runners estimated the residual mass within the cylinder. Temperatures throughout the cycle are calculated using the ideal gas law and the estimated trapped mass. These temperatures are estimated to be accurate to within $+/-5 \%$. Standard heat release analysis was used to determine burn locations during combustion.

\section{MANifOLd Filling Dynamics (MFD)}

Based on the assumption of an isothermal intake manifold with $T_{1}=90$ degrees $\mathrm{C}(363 \mathrm{~K})$, two states are sufficient to characterize the intake manifold: the total intake manifold mass, $m_{1}$, and inert gas fraction, $i_{1}$ :

$$
\begin{aligned}
\frac{d}{d t} m_{1} & =W_{01}+W_{21}-W_{1 c} \\
\frac{d}{d t} i_{1} & =\frac{-W_{01} i_{1}+W_{21}\left(i_{2}-i_{1}\right)}{m_{1}} .
\end{aligned}
$$

The ideal gas law relates the intake manifold pressure $p_{1}$ to the mass by $p_{1}=m_{1} \frac{R T_{1}}{V_{1}}$ where $V_{1}$ is the intake manifold volume. The thermodynamic constants are the difference of specific heats at constant pressure and constant volume $(R$, $\mathrm{kJ} /(\mathrm{kg} \mathrm{K}))$ and the ratio of these specific heats $(\gamma)$. The dependence of these variables on the inert gas fraction has been neglected. We assume here that $\left(p_{1}<p_{0}<p_{2}\right)$ so that all the flows through the valves and orifices are due to the pressure difference between the volumes and the flow from the intake manifold to the cylinder $W_{1 c}$ is a forced flow due to cylinder pumping.

$$
\begin{array}{ll}
\frac{d}{d t} m_{2}= & W_{c 2}-W_{20}-W_{21}-W_{2 c} \\
\frac{d}{d t} i_{2}= & \frac{W_{c 2}\left(i_{e r}-i_{2}\right)}{m_{2}} \\
\frac{d}{d t} p_{2}= & \frac{\gamma R}{V_{2}}\left(W_{c 2} \tilde{T}_{e r}-\left(W_{20}+W_{21}+W_{2 c}\right) T_{2}\right),
\end{array}
$$

Three states represent the gas filling dynamics in the exhaust manifold: mass, $m_{2}$, pressure, $p_{2}$, and inert gas fraction, $i_{2}$ (3). $V_{2}$ is the volume of the exhaust manifold. The temperature of the gas entering the exhaust manifold is $\tilde{T}_{e r}=T_{e r}-\Delta T_{e r 2}$ where $\Delta T_{e r 2}$ is a temperature drop of $75^{\circ} \mathrm{C}$, based on test data, from the exhaust runner temperature $\left(T_{e r}\right)$. The ideal gas law relates the temperature to the pressure and mass values: $T_{2}=p_{2} V_{2} /\left(R m_{2}\right)$. $W_{c 2}$ is the average mass flow rate from the cylinder to the exhaust manifold, calculated as delayed cycle-sampled total flow into the cylinder:

$$
W_{c 2}(t+\tau)=W_{1 c}(t)+W_{f}(t)+W_{2 c}(t) .
$$

The temperature, $T_{e r}$, and inert gas fraction, $i_{e r}$, of the mass flow through the exhaust runner are also calculated via delays:

$$
\begin{array}{r}
T_{e r}(t+\tau)=T_{b d}(t) \\
i_{e r}(t+\tau)=i_{b d}(t)
\end{array}
$$

where $T_{b d}$ and $i_{b d}$ are the temperature and inert gas fraction, respectively, of the blowdown gas into the exhaust runner. These quantities are calculated in the last phase of the combustion model, presented in Sec. VII-C. Again, $\tau$ represents the cycle delay imposed by the engine's cyclic behavior. Note here that the differential equations (2)-(3) can be discretized using the Euler's method on an interval equal to the engine cycle. For higher fidelity we leave the manifold filing dynamics of Eq. (2)-(3) in the continuous-intime domain and interface with the discrete-in-time cylinder delay dylamics of Eq. (4)-(6) with a sample and zero-order hold $(\mathrm{ZOH})$.

The flows $W_{x y}$ from volume $x$ to volume $y$ are based on an orifice flow equation ([7], Appendix C). The EGR flow $W_{21}$, via the EGR valve effective flow area $C_{d} A_{e g r}\left(u_{e g r}\right)$ is determined by $u_{\text {egr }}$.

\section{Cycle-Average Cylinder Flow}

The mean flows into the cylinder, $W_{1 c}$ and $W_{2 c}$ are calculated

$$
\begin{aligned}
& W_{1 c}=\frac{n_{c}\left(1-x_{r}\right)}{n_{c} x_{r}} m_{c}-W_{f} \\
& W_{2 c}=\frac{m_{c}}{\tau}
\end{aligned}
$$

where $n_{c}=1$ is the number of cylinders, $m_{c}$ is the trapped cylinder mass at IVC (derived in the next section), $W_{f}$ is the mean fuel flow rate into all of the cylinders and $x_{r}$ is the mass fraction of internal (higher temperature) residual gases at $\theta_{i v c}$ ([7] p. 102). For the system's fixed intake valve closing angle, $\theta_{i v c}$, and constant intake manifold temperature, $T_{1}, x_{r}$ is parameterized by:

$$
x_{r}=\alpha_{1}\left(1+\kappa_{0} \frac{p_{1}^{\kappa_{1}}}{p_{2}^{\kappa_{2}} \sqrt{T_{e r}}}\right) \underbrace{\left(u_{r b l}+\alpha_{2} u_{r b l}^{2}+\alpha_{3} u_{r b l}^{3}\right)}_{C_{d} A_{r b l}}
$$

where all the $\alpha$ and $\kappa$ coefficients have been identified using experimental data and simulations of a GM proprietary engine model with crankangle-resolved flows as shown in the specific example of Fig. 4 for $u_{r b l}=3 \mathrm{~mm}$.

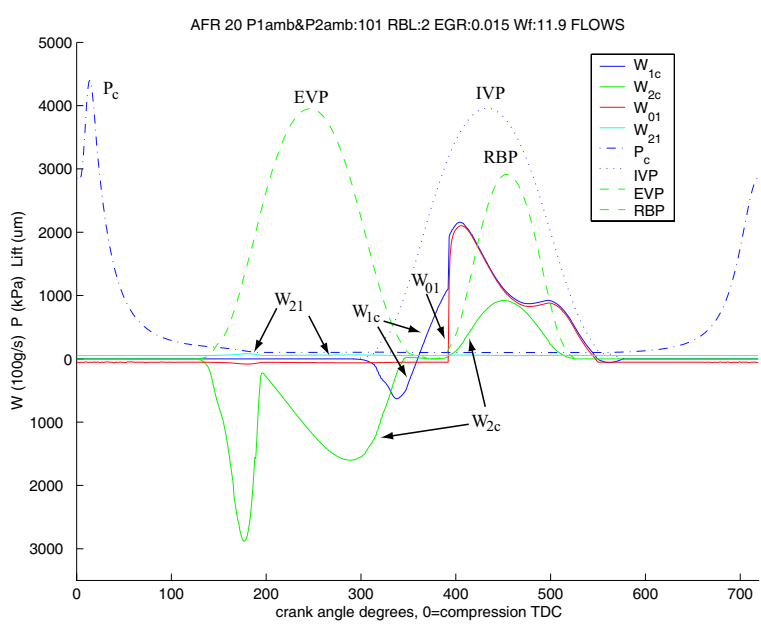

Fig. 4. Cylinder flow and other crankangle-resolved variables. 
A comparison of regressed and experimental cycleaveraged test values for the estimated flows is shown in Fig. 5. Increasing rebreathing lift correlates with a larger value of $x_{r}$, but the sensitivity of $x_{r}$ to $u_{r b l}$ decreases as the rebreathing lift actuator approaches its maximum value of $4 \mathrm{~mm}$ as shown also in Fig. 5. Rebreathing lift effectively throttles the flow through the "pump"-like cylinder.

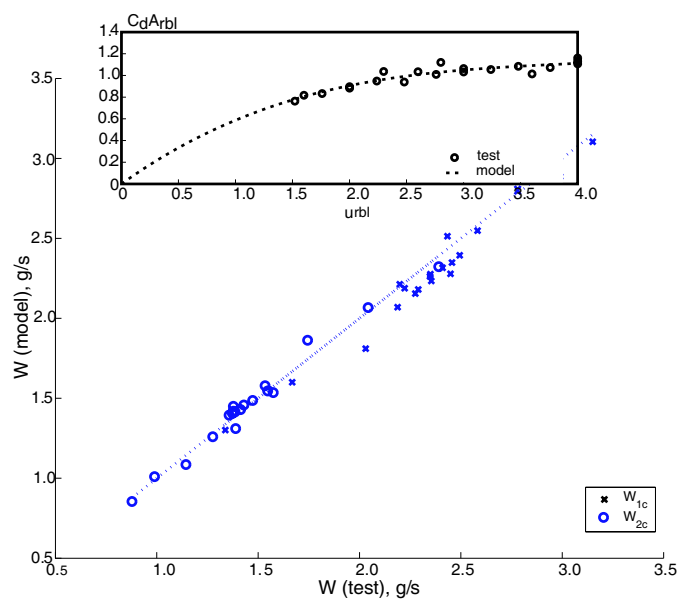

Fig. 5. Validation of predicted mean cylinder flows

Residual gas fraction, $x_{r}$ in the cylinder at IVC, as defined in the preceding paragraphs, is distinct from the inert gas fraction in the cylinder, $i_{c}$, which is calculated below, in (11). Residual gas fraction, $x_{r}$, defines the effect of the mean value model states, $x$, and the rebreathing lift, $u_{r b l}$, on the relative magnitudes of the mass flows, $W_{1 c}$ and $W_{2 c}$, into the cylinder. Inert gas fraction in the cylinder, $i_{c}$, tracks the aggregate percentage of inert gases from these two sources.

\section{Conditions at Intake Valve Closing (IVC)}

We approximate cylinder pressure at IVC as a linear function of $p_{1}$ :

$$
p_{i v c}=\beta_{0}+\beta_{1} p_{1}
$$

The charge temperature at IVC is calculated as the massweighted average of the temperatures of the flows contributing to the trapped mass $m_{c}$ :

$$
\begin{aligned}
T_{i v c} & =T_{1}\left(1-x_{r}\right)+T_{e r} x_{r} \\
m_{c} & =\frac{p_{i v c} V_{i v c}}{R T_{i v c}} .
\end{aligned}
$$

Remaining conditions at intake valve closing that will be required by the combustion model are:

$$
\begin{aligned}
i_{c} & =\left(1-x_{r}\right) \frac{W_{1 c}}{W_{1 c}+W_{f}} i_{1}+x_{r} i_{e r} \\
A F R_{c} & =\frac{\left(1-i_{1}\right) W_{1 c}+\left(1-i_{e r}\right) W_{2 c}}{W_{f}}
\end{aligned}
$$

\section{HCCI COMBUStion MOdeL}

Assuming complete and lean combustion, $i_{b d}$ (13) is unaffected by the combustion process and depends only on two of the conditions at IVC: $i_{c}$ and $A F R_{c}$.

$$
i_{b d}=\frac{A F R_{s}+1}{A F R_{c}+1}\left(1-i_{c}\right)+i_{c}
$$

where $A F R_{s}$ is the stoichiometric air-to-fuel ratio.

Air-to-fuel ratio from an exhaust gas oxygen sensor, $A F R_{E G O}$, is:

$$
\begin{aligned}
& \frac{d}{d t} A F R_{E G O}=\frac{1}{\tau_{E G O}}\left(A F R_{2}-A F R_{E G O}\right) \\
& A F R_{2}=\frac{1-i_{2}+A F R_{s}}{i_{2}} .
\end{aligned}
$$

EGO sensor time constant is $\tau_{E G O}$. This expression is equivalent to an algebraic rearrangement of the expression for $F_{e}\left(i_{2}\right.$ here) as a function of $r_{e}\left(A F R_{2}\right.$ here) in [6].

A simplified combustion model provides the remaining variables, exhaust blowdown temperature, $T_{b d}$, and the performance variable, $\theta_{C A 50}$ as a function of $T_{i v c}$ and $p_{i v c}$. The model consists of five sequential phases: (i) polytropic compression that leads to start of combustion (SOC) through autoignition at $\theta_{s o c}$, (ii) combustion duration that determines an effective $\theta_{C A 50}$, preceding (iii) simulated instantaneous heat release, (iv) polytropic expansion, and (v) adiabatic blowdown that yields $T_{b d}$. These phases are depicted in Fig. 6, where the actual (solid line) and modelled (dotted line) pressure and temperature trajectories are shown.
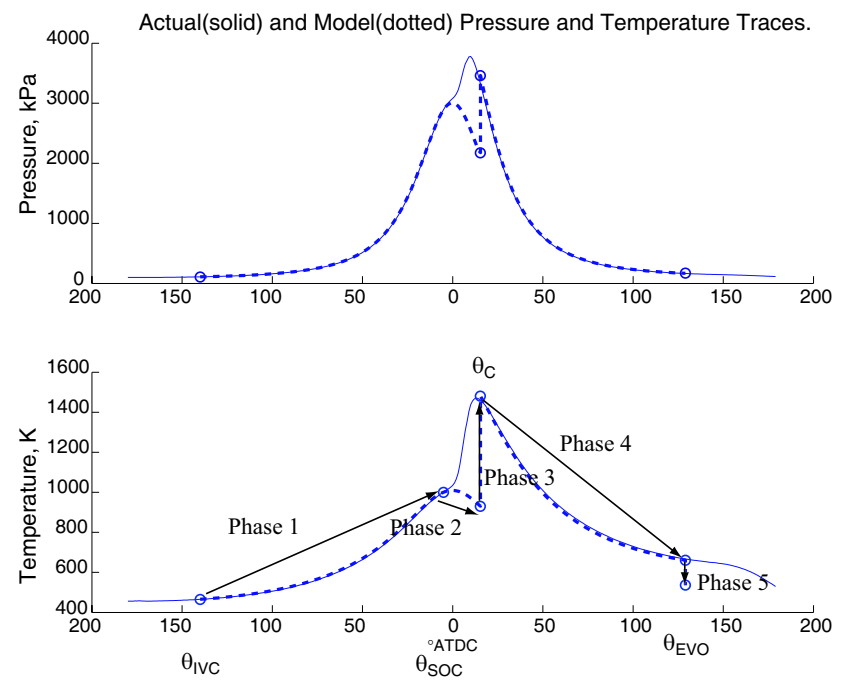

Fig. 6. Actual (solid) and estimated (dotted) pressure and temperature traces in cylinder, from before IVC to after EVO. Calculated model values are indicated with circles. 


\section{A. Phase 1: Intake Valve Closing to Start of Combustion}

Motivated by [9], [1], [17], the Arrhenius integral predicts the SOC timing, $\theta_{S O C}$ :

$$
\begin{array}{r}
A R\left(\theta_{s o c}\right)=1 \quad \text { where } A R(\theta)=\int_{\theta_{i v c}}^{\theta} R R(\vartheta) d \vartheta \\
\text { and } \quad R R(\vartheta)=A p_{c}^{n}(\vartheta) \exp \left(-\frac{E_{a}}{R_{a} T_{c}(\vartheta)}\right)
\end{array}
$$

where $T_{c}$ and $p_{c}$ are cylinder temperature and pressure, respectively, during compression, $A$ is a scaling constant, $E_{a}$ is the activation energy for the autoignition reaction and $\mathrm{n}$ indicates the reaction's sensitivity to pressure. We define $v_{i v c}(\vartheta)=V_{c}\left(\vartheta_{i v c}\right) / V_{c}(\vartheta)$ with $V_{c}(\vartheta)$ the cylinder volume at crankangle $\vartheta$, and assume polytropic compression from IVC to SOC with coefficient $n_{c}$. The Arrhenius integrand is thus simplified

$$
R R(\vartheta)=A p_{i v c}^{n} v_{i v c}^{n_{c} n}(\vartheta) \exp \left(-\frac{E_{a} v_{i v c}^{1-n_{c}}(\vartheta)}{R_{a} T_{i v c}}\right)
$$

and the coefficients $A, E_{a}$ and $n$ are selected using $\theta_{\text {soc }}=$ $\theta_{C A 01}$. With $\theta_{\text {soc }}$ defined by (15)-(17), the charge temperature at SOC is $T_{s o c}=T_{i v c} v_{i v c}^{\left(n_{c}-1\right)}\left(\theta_{s o c}\right)$. The resulting predictions of $\theta_{s o c}$ and $T_{s o c}$ are shown in Fig. 7.
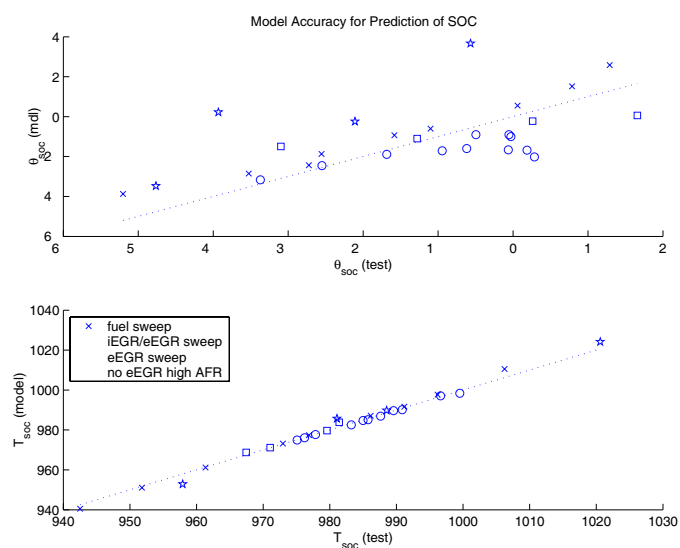

Fig. 7. Plots showing prediction accuracy for $\theta_{s o c}$ and $T_{s o c}$.

\section{B. Phase 2: Combustion Duration}

We define $\mathrm{HCCI}$ combustion duration $\Delta \theta$ as the crankangle degrees between $1 \%$ and $90 \%$ fuel burnt. Heat release due to combustion is considered to occur instantaneously at $\theta_{c}$ [4]:

$$
\begin{array}{ll} 
& \theta_{c}=\theta_{s o c}+\Delta \theta \\
\text { where } & \Delta \theta=k\left(T_{s o c}\right)^{(-2 / 3)}\left(T_{m}\right)^{1 / 3} \exp \left(\frac{E_{c}}{3 R_{c} T_{m}}\right) \\
\text { and } & T_{m}=T_{s o c}+e\left(1-i_{c}\right) \Delta T \\
\text { finally } & \Delta T=\frac{Q_{L H V}}{c_{v}\left(1+A F R_{c}\right)} .
\end{array}
$$

Activation energy, $E_{c}$, represents an effective threshold at which the combustion reaction occurs and is assumed to be $185 \mathrm{~kJ} / \mathrm{mol}$. The parameter $e$ represents an averaging of the released thermal energy during combustion. The parameter $k$ relates to the effective combustion duration,
$\Delta \theta$, during which no heat release is modeled. As duration lengthens, a smaller proportion, $e$, of the released heat contributes to an effective mean temperature, $T_{m}$ during the combustion. After the combustion duration, instantaneous heat release occurs at the combustion location, $\theta_{c}$. Both of the parameters $k$ and $e$ have been optimized to match the actual burn duration from $1 \%$ to $90 \%$. Crank angle of $50 \%$ burnt fuel is calculated as $\theta_{C A 50}=\theta_{s o c}+.55 \Delta \theta$.

Since $e$ is a measure of the average temperature during combustion, a lower value of $k$ corresponds to a shorter combustion, which also corresponds to a higher value for $e$. The combinations of independently optimized values of $k$ and $e$ results in a single relation (19) for which the value for $e$ is uniquely determined by the value for $k$

$$
e=a_{0}+a_{1} k
$$

This yields a physically-based combustion duration model whose variations over the operating range can now be captured with a single parameter, $k$. Optimized values of $k$ that precisely reproduce the combustion duration for each set of test data are then parameterized as a function of $\theta_{s o c}$ :

$$
k=b_{0}+b_{1} \theta_{s o c}+b_{2} \theta_{s o c}^{2} .
$$

The accuracy of these predictions can also be seen in Fig. 8 .

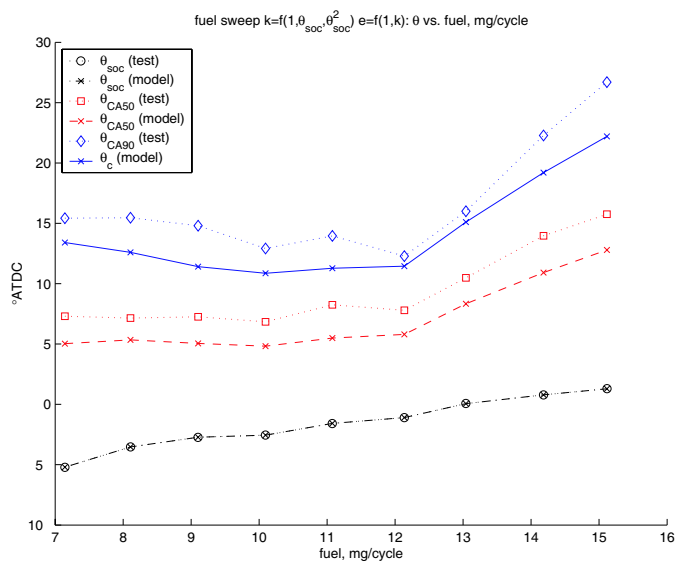

Fig. 8. Plot showing the impact of the parameterized values for $\mathrm{k}$ on the burn duration for the fuel sweep data.

\section{Phase 3: Instantaneous Heat Release}

The charge temperature just before the combustion can be calculated by $T_{b c}=T_{i v c} v_{i v c}^{\left(n_{c}-1\right)}\left(\theta_{c}\right)$ and the corresponding pressure as $p_{b c}=p_{i v c} v_{i v c}^{n_{c}}\left(\theta_{c}\right)$. Assuming an instantaneous release of all of the fuel's heat, the charge temperature and pressure after the combustion are given by:

$$
T_{a c}=T_{b c}+\left(1-i_{c}\right) \Delta T \text { and } p_{a c}=p_{b c} T_{a c} / T_{b c}
$$

D. Phase 4: Polytropic Expansion

To account for the polytropic expansion with coefficient $n_{e}$, a volume ratio analogous to $v_{i v c}(\vartheta)$ is defined as $v_{c}(\vartheta)=V_{c}\left(\vartheta_{c}\right) / V_{c}(\vartheta)$ so that the charge temperature and pressure at EVO is

$$
T_{\text {evo }}=T_{a c} v_{c}^{\left(n_{e}-1\right)}\left(\theta_{\text {evo }}\right) \text { and } p_{\text {evo }}=p_{a c} v_{c}^{n_{e}}\left(\theta_{\text {evo }}\right) .
$$




\section{E. Phase 5: Exhaust Blowdown}

After EVO, exhaust temperature leaving the cylinder can be considered as

$$
T_{b d}=T_{\text {evo }} \frac{p_{2}}{p_{\text {evo }}} \frac{n_{e}-1}{n_{e}}+\Delta T_{b d}
$$

due to the adiabatic expansion of the gas down to the exhaust manifold pressure, $p_{2}$, offset by a constant temperature difference, $\Delta T_{b d}$.

The model provides a minimal mathematical representation of the physical combustion process as well as a basis function based for which the six parameters, $A, E_{a}$, and $n$ from (17) and $k, E_{c}$ and $e$ from (21) can be tuned to fit all available data in the future. The $\theta_{s o c}$ and $\theta_{C A 50}$ predictions are shown in Fig. 9. The $T_{b d}$ predictions of the HCCI Combustion model are shown in Fig. 10.

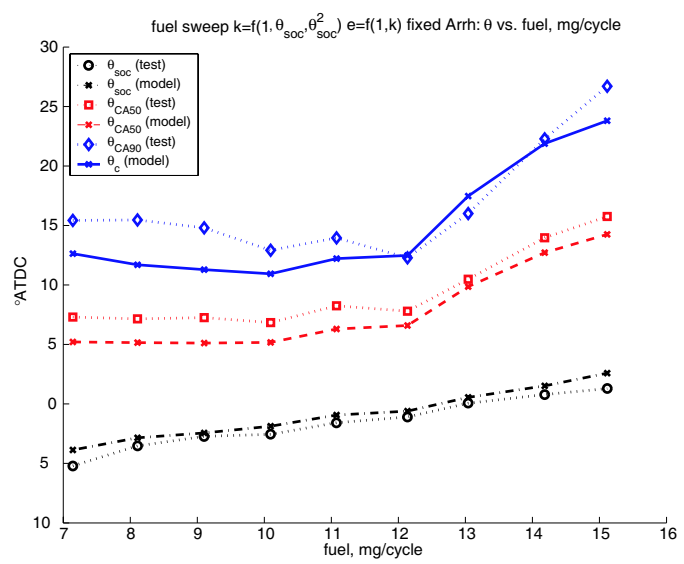

Fig. 9. Comparison of integrated combustion model predictions of $\theta_{s o c}$ and resulting predictions of $\theta_{C A 50}$ with test data for fuel sweep.

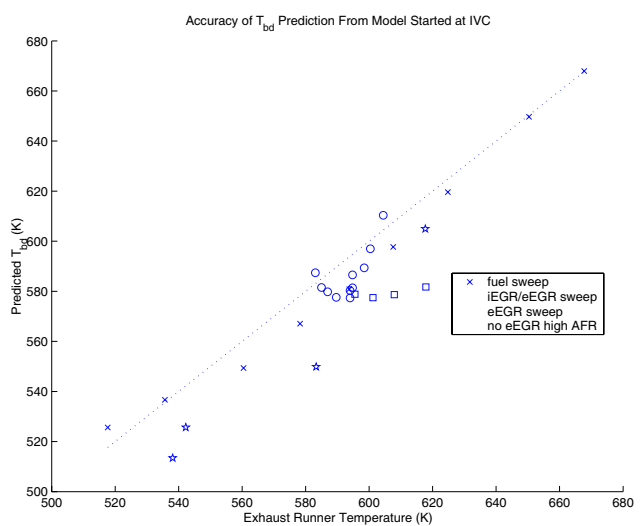

Fig. 10. Exhaust runner blowdown temperature prediction based on all five phases of the model from conditions at intake valve closing.

\section{VALIDATION}

The HCCI engine model combines the manifold filling dynamics of Sec. IV with the simplified combustion model presented in Sec. VII as shown in Fig. 3. Though the MFD and HCCI models were separately validated, the validation of the integrated model is important because the temperature at the end of the combustion (product of the HCCI combustion model) affects the thermodynamic state (pressure, temperature) of the next combustion event through the manifold filling dynamics (MFD model). The high dilution of this HCCI engine increases the significance of this internal feedback that generally exists to a smaller extent in conventional spark-ignition and compressionignition internal combustion engines.

The orifice areas, $C_{d} A_{01}, C_{d} A_{20}$, and $C_{d} A_{21}\left(u_{e g r}\right)$ are calibrated using ambient condition $p_{0}=101 \mathrm{kPa}$. The remaining inputs, $u_{r b l}$, and the fueling rate, $W_{f}$ are the constant commanded values from the tests.

The steady-state flows are shown in Fig. 11. Due to the light throttle conditions, a deviation in $p_{1}$ causes noticeable deviation in $W_{01}$. The maximum error appears in the case of the $11 \mathrm{mg} / \mathrm{cycle}$. Predictions of $T_{i v c}$ show good accuracy, with the $11 \mathrm{mg}$ point showing the greatest overprediction, as shown in Fig. 12.
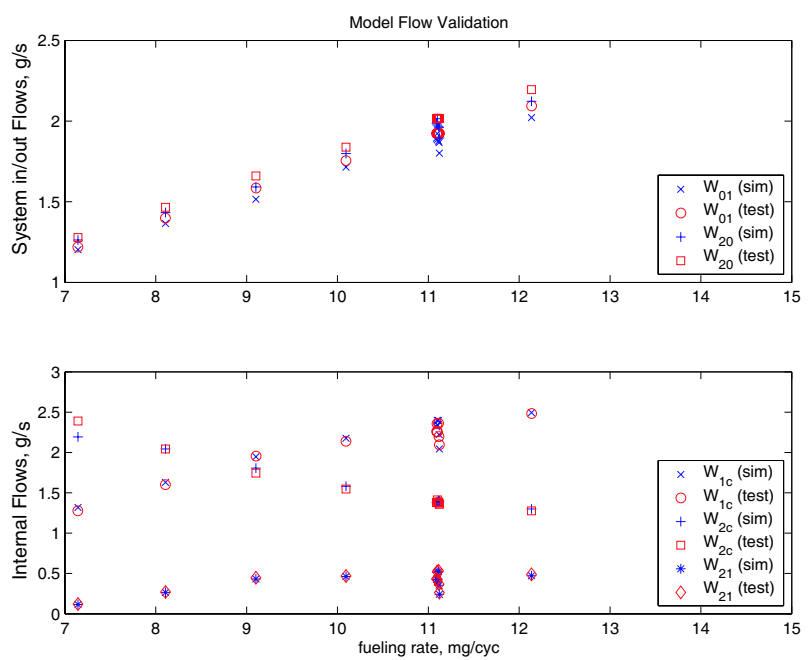

Fig. 11. Actual and simulated flows for the integrated combustion model using ambient pressure of $101 \mathrm{KPa}$ connected to both intake and exhaust manifolds.

Finally, consistent with reduced $W_{01}$ for the $11 \mathrm{mg} /$ cycle point shown in Fig. 11, the prediction of $A F R$ at this operating point is lower, 19.5 versus 20 for the actual test data. This discrepancy in $A F R$ prediction is shown in Fig. 13. Also, the higher value for $T_{i v c}$ leads to a prediction of earlier $\theta_{s o c}$ and performance variable $\theta_{C A 50}$.

\section{CONCLUSiOns}

We present a simple mean value model and validate it in steady-state. Despite some discrepancies in the integrated model steady-state predictions, the trends in flows, states, conditions at IVC, and performance parameters are reproduced. Specifically, the model captures:

- the intake and exhaust manifold flows and the effects of the external and internal EGR

- the modulating effects of the actuators, $u_{e g r}$ and $u_{r b l}$ 

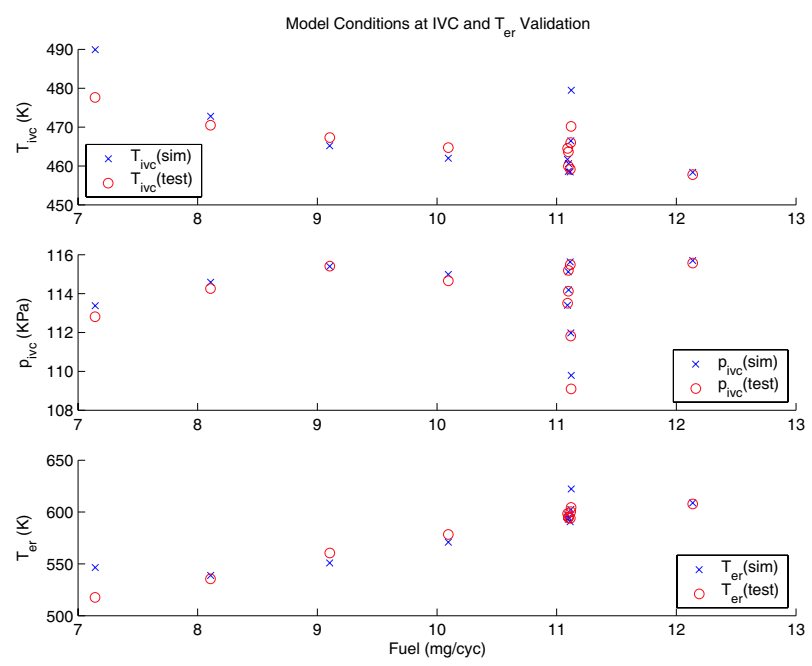

Fig. 12. Actual and simulated conditions at IVC, $T_{i v c}$ and $p_{i v c}$ and exhaust runner temperature, $T_{e r}$.
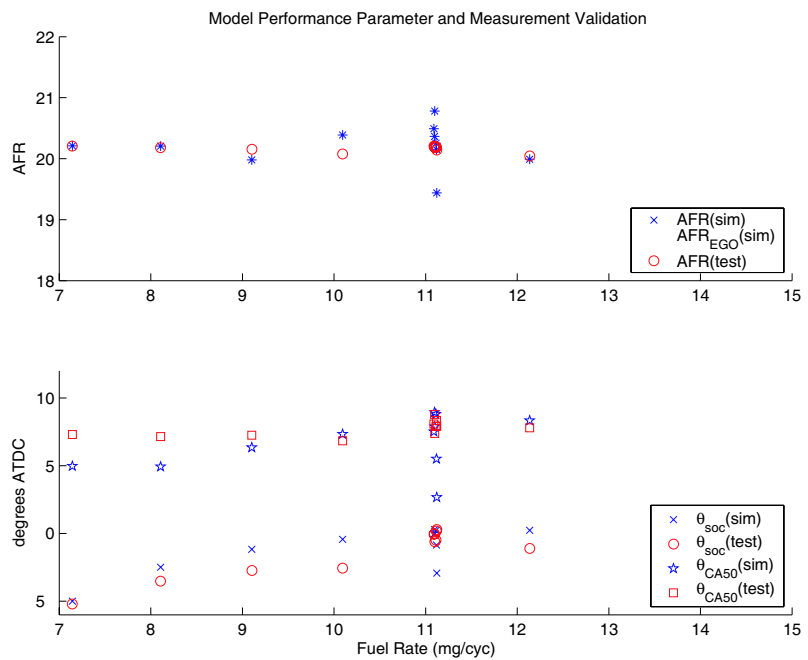

Fig. 13. Actual and simulated performance parameters and related measurements.

- the impact of the system state variables and actuators on the performance variable, conventional $A F R$ (inlet mass air flow rate/fuel flow rate), the tailpipe measured value, $A F R_{E G O}$, and the actual in-cylinder air-fuel ratio, $A F R_{c}$

- the impact of the system state variables and actuators on the performance variable $\theta_{s o c}$ and $\theta_{C A 50}$

The model includes three continuous manifold states, three discrete cylinder states and one sensor lag. Though the existing experimental setup did not allow validation of transient behavior, the model can provide the basis for dynamical analysis and feedback control design of the HCCI engine.

\section{ACKNOWLEDGments}

We thank Sharon Liu, Jason Chen and Man-Feng Chang of General Motors Corporation, George Lavoie of the
University of Michigan, and Paul Ronney of the University of Southern California.

\section{REFERENCES}

[1] Agrell F. Angstrom H.-E., Eriksson B., Linderyd J., "Integrated Simulation and Engine Test of Closed Loop HCCI Control by aid of Variable Valve Timing," SAE paper 2003-01-0748.

[2] J. Bengtsson, P. Strandh, R. Johansson, P. Tunestal, B. Johansson, "Cycle-To-Cycle Control of a Dual-Fuel Hcci Engine," SAE paper 2004-01-0941.

[3] J. C. Cantor, "A Dynamical Instability of Spark-Ignited Engines," Science, Vol 224(4654), pp. 1233-1235. June 14, 1984

[4] C. Ji, P. D. Ronney, "Modeling of Engine Cyclic Variations by a Thermodynamic Model," SAE 2002-01-2736.

[5] Martinez-Frias J., Aceves S. M., Flowers D., Smith J. R., Dibble R., "HCCI Control by Thermal Management," SAE paper 2000-01-2869.

[6] J. Grizzle, J. Buckland and J. Sun, "Idle speed control of a direct injection spark ignition stratified charge engine", Int. J. of Robust and Nonlinear Control, (11)pp.1043-71, Sept2001.

[7] Heywood, J.B., Internal Combustion Engine Fundamentals, McGrawHill, Inc., 1988.

[8] Kontarakis G., Collings N., Ma T., "Demonstration of HCCI Using a Sinlge Cylinder Four-stroke SI Engine with Modified Valve Timing," SAE paper 2000-01-2870.

[9] Livengood, J.C., Wu, P.C. "Correlation of Autoignition Phenomena in Internal Combustion Engines and Rapid Compression Machines," Fifth Symposium on Combustion, 1955.

[10] Najt P., Foster D., "Compression-Ignited Homegeneous Charge Combustion," SAE paper 830264.

[11] Olsson J. O., Tunestal P., Johansson B., "Closed Loop Control of an HCCI Engine," SAE paper 2001-01-1031.

[12] Olsson J. O., Tunestal P., Johansson B., Fiveland S., Agama R., Willi M., Assanis D., "Compression Ratio Influence on Maximum Load of a Natural Gas Fueled HCCI Engine," SAE paper 2002-01-0111.

[13] Rausen, D. J. "A Dynamic Low Order Model of Homogeneous Charge Compression Ignition Engines," MS Thesis, Mechanical Engineering, The University of Michigan, June 2003.

[14] G.M. Shaver, J.C. Gerdes, P. Jain, P.A. Caton, C.F. Edwards, Modeling for Control of HCCI Engines, Proc. of the American Control Conf., pp. 749-754, 2003.

[15] G.M. Shaver and J.C. Gerdes, Cycle-to-cycle control of HCCI Engines, 2003 ASME Proc. of International Mechanical Engineering Congress and Exposition IMECE2003-41966.

[16] Stanglmaier D. S., Roberts E., "Homogenous Charge Compression Ignition (HCCI): Benefits, Compromises, and Future Engine Applications," SAE paper 1999-01-3682.

[17] Willand J., Nieberding R.-G., Vent G., Enderle C., "The Knocking Syndrome - Its Cure and Its Potential”, SAE paper 982483. 